Gut, 1980, 21, 195-201

\title{
Inflammatory bowel disease associated circulating immune complexes*
}

\author{
B J KEMLER† AND E ALPERT
}

From the Medical Service (Gastrointestinal Unit), Massachusetts General Hospital and the Department of Medicine, Harvard Medical School, Boston, Massachusetts, USA

SUMMARY Circulating immune complexes have been detected in patients with inflammatory bowel disease (IBD). To determine if these complexes are related specificially to IBD or more generally to loss of intestinal mucosal integrity, we compared circulating immune complex levels in the sera of 86 IBD patients, nine pseudomembranous and nine bacterial colitis patients, and 42 healthy controls. Immune complexes were measured by a Raji cell radioimmunoassay. Raji detectable circulating immune complex levels were significantly higher in the IBD group than in the healthy controls $(P<0.001)$. Circulating immune complex levels in the pseudomembranous-bacterial colitis group and the healthy controls were essentially identical. While nearly $20 \%$ of the IBD patients (16 of 86 ) had abnormally high levels, none of the patients with the other forms of intestinal inflammation $(0$ of 18$)$ had abnormal levels. These data suggest that the circulating immune complexes present in inflammatory bowel disease patients are related to the IBD process rather than to non-specific mucosal cell (barrier) damage. Patients with intestinal inflammation and normal peripheral immune complex levels also had normal mesenteric vein levels. These data suggest that lack of formation, rather than more efficient hepatic reticuloendothelial clearance, was primarily responsible for the absence of detectable complexes in Raji negative individuals. Circulating immune complex levels did not correlate with type, location, severity, or extraintestinal manifestations of inflammatory bowel disease. The absence of Raji detectable circulating immune complexes in the majority of patients, even in those with extraintestinal manifestations, raises serious doubts about the pathogenic significance of such complexes. Nevertheless, as the circulating immune complexes appear to be disease related, they may be used to isolate and identify disease specific antigen(s) of possible aetiological importance.

Circulating immune complexes (CIC) have been detected in the sera of certain patients with inflammatory bowel disease (IBD), using a variety of indirect techniques. ${ }^{1-6}$ The multiplicity of assay systems which have indicated raised levels in 20 to $40 \%$ of IBD patients supports the interpretation that the reacting substances are immune complexes and not endotoxin, DNA, or similar interfering compounds. Despite the extensive data documenting the existence of CIC in selected patients with IBD, it is uncertain whether such complexes are specific for idiopathic inflammatory bowel disease(s) or are secondary to non-specific disruption of the mucosal barrier. The uncertainty stems from the failure of

*This work was supported in part by grants from the National Foundation for Ileitis and Colitis and the NIH (AM07191).

†Address for reprint requests: Dr Barry Kemler, Gastrointestinal Unit, Massachusetts General Hospital, Boston, Massachusetts 02114 , USA.

Received for publication 16 October 1979 previous investigators to examine patients with other forms of intestinal inflammation resulting in mucosal denudation for the presence of CIC.

A determination of whether the circulating complexes are related to loss of mucosal integrity in general, or to inflammatory bowel disease in particular, is of considerable importance. A demonstration that circulating immune complexes occur with non-specific inflammation would suggest strongly that they are not of pathogenic significance in IBD. On the other hand, the demonstration that such complexes are related specificially to idiopathic inflammatory bowel disease, while not necessarily denoting pathogenic significance, would indicate that the complexes may be employed to isolate, characterise, and identify disease specific antigens. Such antigens, even in non-pathogenic complexes, may prove to be of aetiological or diagnostic significance.

In the present study the relationship of circulating 
immune complexes to inflammatory bowel disease was examined by comparing levels present in the sera of IBD patients with levels present in the sera of patients having prolonged active pseudomembranous or bacterial colitis. A sensitive Raji cell radioimmunoassay was used to detect complexes. The relationship between CIC levels and disease status, and the role of the hepatic reticuloendothelial system in determining the presence of peripheral circulating immune complexes were also examined.

\section{Methods}

Eighty-six patients with inflammatory bowel disease comprised one study group (group I). Thirty-two ulcerative colitis (UC), 11 granulomatous colitis (GC), 16 granulomatous ileocolitis (GIC), and 27 regional enteritis (RE) patients were included in this group, obtained from the inpatient and outpatient adult medical services of the Massachusetts General Hospital. The diagnosis of IBD was based upon the clinical history, contrast $x$-ray examinations, endoscopy, and histology of surgical or biopsy specimens. All patients included in the study had findings typical of their disease by at least three of these criteria. Thirty patients had mild, 32 had moderate, and 24 had severe disease as determined by criteria established by Truelove et al. ${ }^{7}$ and de Dombal et al. ${ }^{8}$ for ulcerative colitis and Crohn's disease, respectively. Twenty-two of the 86 IBD patients had extracolonic manifestations of their disease; including nine with arthritis or arthralgias, six with biochemical evidence of pericholangitis, four with cutaneous lesions, and three with uveitis. Steroids alone, azulfidine alone, a combination of steroids and azulfidine, or neither medication was received by $25,20,14$, and 27 patients, respectively. Eighteen patients with 'non-IBD' colitis comprised a second study group (group II). Nine patients with pseudomembranous colitis (sera and clinical data obtained from Dr John Bartlett of the Boston VA) and nine patients with severe bacterial colitis seen at this hospital were included in group II. The clinical diagnosis of pseudomembranous colitis was confirmed in each patient by sigmoidoscopy and the specific in vitro neutralisation, by Clostridium sordellii antitoxin, of stool extract induced tissue cytopathic effects. ${ }^{9}$ These studies were kindly performed by Dr John Bartlett. All cases of bacterial colitis were confirmed by positive cultures for known pathogens. Severe disease was defined by more than six stools/day, rectal bleeding, and systemic symptoms including a temperature higher than $100^{\circ} \mathrm{F}$ and pulse greater than $90 /$ minute. All the pseudomembranous colitis patients had documented disease for more than 10 days and patients with bacterial colitis had active disease for approximately one week.

Forty-two healthy laboratory workers and hospital personnel comprised the control group (group III). The sex and age distributions were comparable in the control and IBD groups. Informed consent was obtained from all participants in the study.

Five millilitres of peripheral venous blood were collected from each participant. In addition, $5 \mathrm{ml}$ of mesenteric venous blood were also obtained from 11 IBD patients undergoing surgical resection of diseased bowel. Blood samples were allowed to clot for one hour at room temperature and the serum removed, frozen, and stored at $-70^{\circ} \mathrm{C}$ before testing.

Serum immune complexes were measured by a modification of the standard Raji cell radioimmunoassay. ${ }^{10}$ In brief, $25 \mu$ l of the test serum (diluted 1:4) were incubated with $3 \times 10^{6} \mathrm{Raji}$ cells at $37^{\circ} \mathrm{C}$ for 45 minutes, followed by washing (three times) with Hank's buffered salt solution lacking calcium or magnesium. The washed cells were incubated with $25 \mu \mathrm{I}^{125}$ I-labelled rabbit anti-human IgG (containing $15 \mu g$ rabbit IgG), at $4^{\circ} \mathrm{C}$ for 30 minutes. The cells were washed and the amount of ${ }^{125} \mathrm{I}$ bound to the Raji cells was determined by scintillation spectrometry. Antihuman IgG was prepared by immunisa-

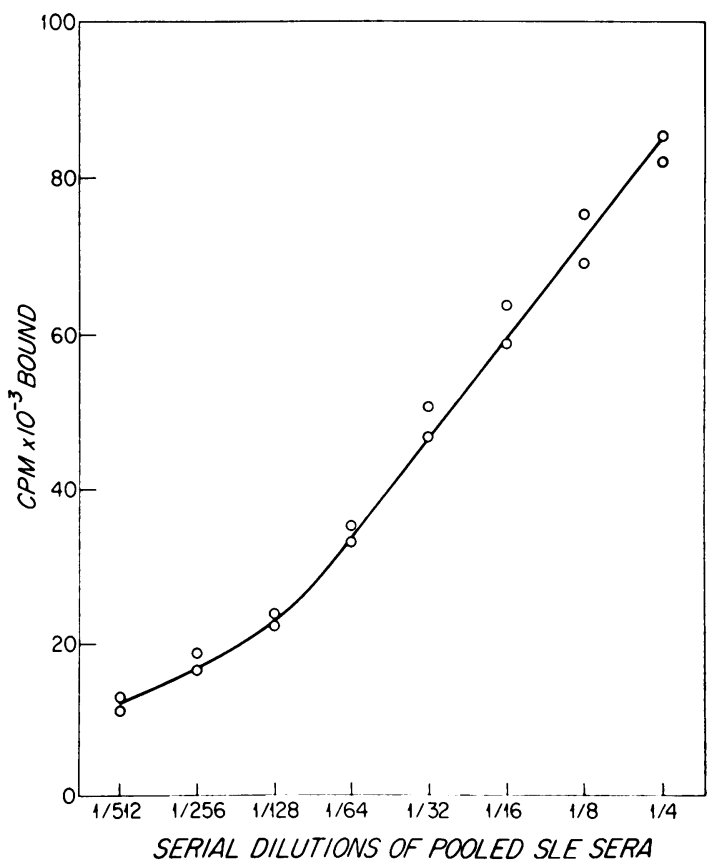

Fig. 1 A typical Raji cell radioimmune assay standard curve. The standard curve for each experiment represents the best fit for two separate dilutional series. The interassay $\mathrm{CV}$ is less than $10 \%$. 
tion with purified IgG (Pentex). When reacted against normal human serum, only an IgG precipitin line could be demonstrated on immunoelectrophoresis. The standard curve was constructed with serial dilutions of a pool of sera from patients with systemic lupus erythematosus (SLE) previously shown to contain high levels of circulating immune complexes, rather than dilutions of heataggregated human gamma globulin (AHG). The former yielded standard curves of greater precision at lower levels and greater interassay reproducibility. The coefficient of variation was less than $10 \%$. A standard curve is shown in Fig. 1. 'SLE units' were established by arbitarily assigning a value of 10 SLE units per $\mathrm{ml}$ of serum to the mean CIC level of 42 healthy controls. Values greater than 17.6 SLE units per $\mathrm{ml}$ (two standard deivations (SD) above the normal mean) were considered abnormal. SLE units cannot be directly equated with AHG $\mu \mathrm{g} / \mathrm{ml}$ equivalents because the binding slopes of the two 'standards' are not precisely parallel. Patients with primary biliary cirrhosis and rheumatoid factor positive rheumatoid arthritis, two diseases previously shown to produce circulating immune complexes, ${ }^{1112}$ served as controls to validate the assay.

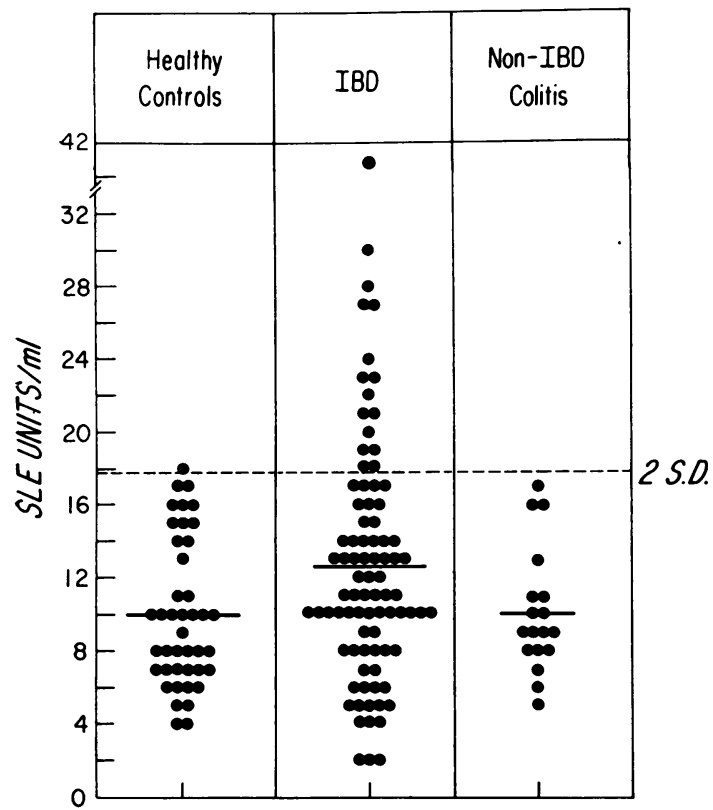

Fig. 2 CIC levels in 42 healthy controls, 86 patients with IBD, and 18 patients with non-IBD colitis-that is, pseudomembranous or bacterial colitis. Values above the broken line (2 SD above the healthy control mean) are abnormal. CIC levels in the IBD group are significantly higher $(\mathrm{P}<0.001)$ than in healthy controls or non-IBD colitis patients.
Eighty-four percent of 32 PBC patients and $100 \%$ of 10 rheumatoid arthritis patients with high RF titres were positive by this assay.

The levels of circulating immune complexes in sera from patients with inflammatory bowel disease, other forms of active colitis, and healthy controls were compared using Student's $t$ test and the Wilcoxon rank sum test. The relationship of CIC to extraintestinal manifestations and the effects of therapy were examined by Chi square analysis.

\section{Results}

CIC IN PERIPHERAL BLOOD OF IBD

PATIENTS AND CONTROLS

The levels of circulating immune complexes in each of the patients and the controls are depicted in Fig. 2. These levels for the IBD group, $12 \cdot 6 \pm 0 \cdot 7$ (mean \pm SEM), were significantly higher than the levels for the healthy control group, $10 \cdot 0 \pm 0 \cdot 6$, by Student's $t$ test and Wilcoxon rank sum analysis $(\mathrm{P}<0.001)$. Although many IBD patients had CIC levels within the normal range, 16 of the 86 patients $(18.6 \%)$ had values above the upper limit of normal. Fractionation of a representative positive serum, by sucrose density gradient ultracentrifugation, demonstrated Raji reactivity sedimenting at approximately 14-15S.

The levels of circulating immune complexes in the nine patients with pseudomembranous colitis and the nine patients with bacterial colitis are also depicted in Fig. 2. The mean CIC level in these 'nonIBD' colitis patients $(10 \cdot 1 \pm 0 \cdot 8)$ was essentially identical with that of the healthy controls, and none of these patients had levels above normal.

CIC IN MESENTERIC VENOUS BLOOD OF IBD PATIENTS

Blood samples were obtained simultaneously from peripheral veins and mesenteric veins draining involved areas in 11 IBD patients at the time of surgical exploration and resection. Figure 3 indicates that the levels of CIC in mesenteric blood are essentially indentical with those found in the peripheral circulation. No significant 'step-down' in Raji detectable CIC occurred across the hepatic RE system in these patients.

IBD STATUS AND IMMUNE COMPLEX LEVELS The inflammatory bowel disease (IBD) group was subdivided into those patients with ulcerative colitis (UC), granulomatous colitis (GC), granulomatous ileocolitis (GIC), and regional enteritis (RE) in order to determine if a particular subset of IBD patients might have raised levels of CIC. Although the ulcerative colitis population $(14 \cdot 2 \pm 0.9)$ was the only subgroup to have statistically significantly 


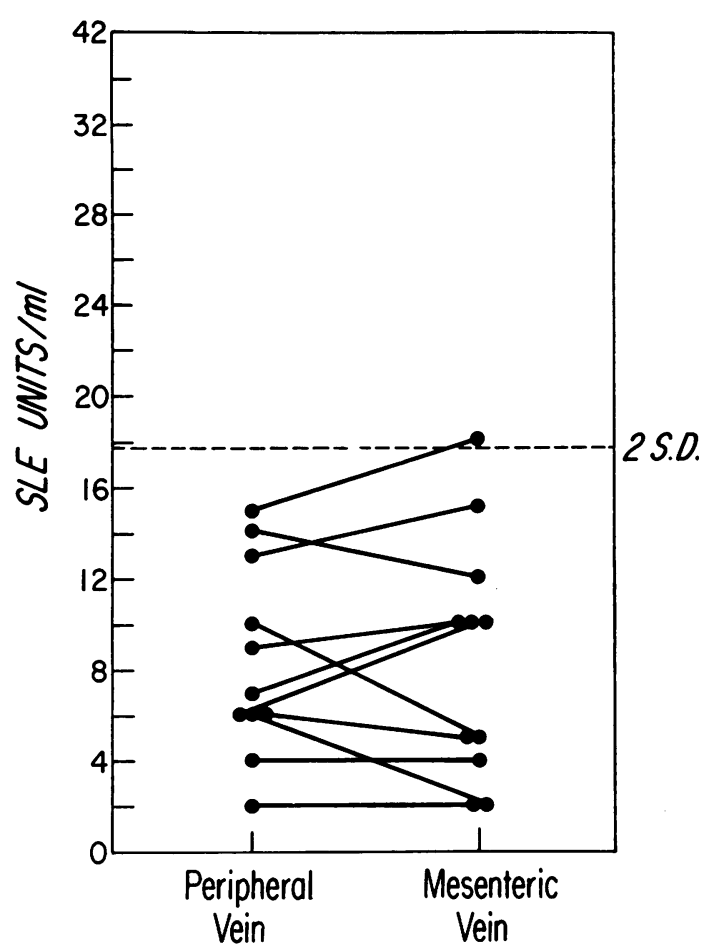

Fig. 3 CIC levels in peripheral and mesenteric venous blood of IBD patients undergoing surgical resections. Patients with normal peripheral blood levels (less than $2 S D$ above the normal mean level of $10 S L E$ units $/ \mathrm{ml}$ ) also have normal mesenteric blood CIC levels.

higher levels than normal $(\mathrm{P}<\mathbf{0 . 0 0 1})$, the mean CIC level in each subgroup was greater than in the healthy control group, and in each subgroup there were some patients with abnormally high levels. Among the three groups of Crohn's disease patients, the Crohn's colitis group had the highest mean level and greatest percentage of patients with abnormal values.

The IBD group was also subdivided on the basis of disease severity - that is, mild, moderate, and severe. As seen in Fig. 4, there was no clustering of patients with abnormal C.IC values in the 'severe disease' category. Although abnormal levels occurrd more frequently in the 'mild' and 'moderate' disease groups, these differences did not reach statistical significance. Ten patients were studied serially. No consistent alteration in CIC levels was observed with change in disease activity. The lack of correlation between disease activity and CIC levels applied to both Crohn's disease and ulcerative colitis patients. Corticosteroid treatment and serum immunoglobulin levels, likewise, did not correlate with ClC levels.

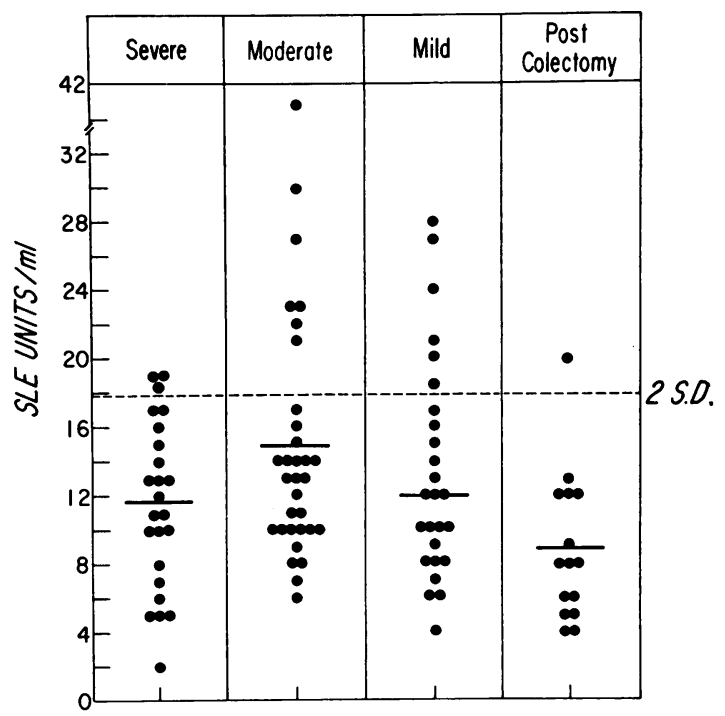

Fig. 4 CIC levels in IBD of varying severity. The one patient in the post-colectomy group with an abnormal CIC level (greater than 2 SD above the normal mean) had a coincident sternal abscess.

Eleven patients were receiving parenteral hyperalimentation and were without oral intake for one week or longer before blood sampling. Three of these patients had raised levels of Raji detectable circulating immune complexes.

Seventeen IBD patients had resection of diseased portions of their intestine and bloods were obtained two weeks after surgery in 15 cases. The mean CIC level in the peripheral sera of this group was identical with the healthy control mean. Only one of the patients had an abnormally high post-resection value (Fig. 4) and that patient had a coexistent corynebacterium sternal abscess.

The mean CIC levels of the inflammatory bowel disease patients with extraintestinal manifestations $(13.9 \pm 1.4)$ was significantly above that of the healthy controls $(10 \cdot 0 \pm 0.6)$, but was not different from the total IBD group. Extraintestinal disease was present in six of 16 patients (38\%) with abnormal CIC values and in 16 of 70 patients $(23 \%)$ with normal values. The slightly higher incidence of complications in patients with raised CIC levels did not reach statistical significance $\left(x^{2} 1.47, \mathrm{P}>0.20\right)$. Of the 16 patients with raised levels of circulating immune complexes levels eight were receiving steroids; only one of the latter group had manifestations of extraintestinal disease, while five of those not receiving steroids displayed extraintestinal manifestations. Despite the significant decrease in expression of extraintestinal disease in all patients receiving steroids $\left(x^{2} 4.26, P<0.05\right)$, exclusion of patients 
receiving steroids from analysis did not result in a significantly higher incidence of extraintestinal disease in the CIC positive group as compared with patients with normal values $\left(x^{2} 0.25\right.$, P $>0.70)$. Furthermore, $73 \%(16 / 22)$ of the patients with extraintestinal disease of various types had normal CIC. A similar distribution of normal to abnormal CIC values occurred with most types of extraintestinal manifestations.

\section{Discussion}

Circulating immune complexes have been detected in patients with inflammatory bowel disease ${ }^{1-6}$. We compared circulating immune complex levels in patients with inflammatory bowel disease with levels in patients having prolonged active pseudomembranous or bacterial colitis, to determine if complexes are related specifically to IBD or more generally to loss of intestinal mucosal integrity.

Our data show that, while the mean CIC level for the IBD group was significantly higher, the mean level for the pseudomembranous and bacterial colitis group was essentially identical with that obtained for the healthy control population. Furthermore, while nearly $20 \%$ of the IBD patients, including those without oral intake, had values above the normal range none of the patients with pseudomembranous or bacterial colitis had abnormal levels. These data strongly suggest that the rises in Raji detectable CIC are related to IBD rather than to a non-specific loss of mucosal integrity.

Although the duration of the mucosal defects in the IBD and patient control groups were dissimilar, this difference probably does not account for the disparity in immune complex levels. All the pseudomembranous colitis patients had documented disease for 10 days or longer and the bacterial colitis patients had active disease for approximately one week. Based on experiments in animals suggesting that peak circulating immune complexes occur between the seventh and 10th day of repeated antigen exposure ${ }^{13}$ it seems probable that the control group would have had sufficient time to allow CIC formation, if production of complexes were solely the result of repeated exposure to luminal antigens. In addition, there was no discernable correlation between the duration of IBD and the presence of CIC abnormalities.

A previous investigation was able to demonstrate large circulating immune complexes only in those IBD patients having concomitant liver disease. ${ }^{3}$ These data have raised the possibility that raised peripheral blood levels reflect a failure of certain IBD patients to clear immune complexes generally entering the portal system secondary to mucosal damage, rather than the formation of specific complexes in IBD. In our study, however, 13 of 16 IBD patients with raised CIC had normal liver function tests. Furthermore, the normal mesenteric vein levels in 11 patients with documented intestinal inflammation suggest that Raji detectable immune complexes do not routinely enter the portal system as a consequence of bowel inflammation and that peripheral levels reflect differential production and not hepatic clearance.

Many investigators have felt that CIC have been detected previously in only a fraction of IBD patients because of the relative insensitivities of the assays used to measure such complexes. In the present investigation, we used a sensitive Raji cell radioimmunoassay capable of detecting a wide range of complement fixing complexes with greater sensitivity than many other complement-dependent systems. ${ }^{14}{ }^{15}$ The detection of immune complexes in the peripheral circulation in only $20 \%$ of the IBD patients studied with his more sensitive assay suggests that assay sensitivity, if it is involved, is not the sole factor responsible for the inability to detect $\mathrm{CIC}$ in more patients with inflammatory bowel disease.

Occurrence of immune complexes may be underestimated because indirect assays for circulating immune complexes generally measure limited subpopulations of complexes. ${ }^{16}$ Incomplete overlap of these subpopulations ${ }^{34}$ means that the number of patients positive for CIC by one assay are partially additive to those positive by another indirect technique. Additionally, underestimation of the frequency of CIC in IBD may result from examination for complexes at a single time point in the course of disease. Relative antigen/antibody concentrations, critical in the detection of complexes, are likely to fluctuate through the course of the illness.

Immune complexes administered to rabbits intravenously have been shown to localise in traumatically inflamed intestinal mucosa. ${ }^{17}$ This observation has led to the hypothesis that IBD may involve formation of CIC in response to 'non-intestinal' antigen(s), and preferential deposition of these complexes in intestinal tissue made susceptible by non-specific inflammation. If this were the case, one would expect resection of the inflamed bowel to result in raised CIC levels. In the present study, only one of the IBD patients had raised levels after a resection, and a coexistent sternal abscess was believed to be the source of the CIC in that patient. Similarly, Fiasse et al. ${ }^{6}$ found the lowest incidence of $\mathrm{CIC}$ in their group of patients with bowel resections. Both findings implicate the bowel as the source rather than the site of deposition of systemically formed complexes. 
Comparison of CIC levels found in groups of patients with different disease activity and examination of levels in individual patients during different stages of disease failed to demonstrate a correlation between disease severity and immune complex levels. Although some studies have reported a correlation between CIC levels and changes in disease status in individual patients, most studies have been generally unsuccessful in demonstrating statistically significant relationships between CIC levels and severity of disease. ${ }^{24}$ Such a relationship does not appear to be masked by the more frequent use of steroids in severe cases, as our data show no correlation between steroid administration and CIC levels. The failure of peripheral CIC levels to correlate with disease activity does not necessarily preclude pathogenic significance for such complexes. The absence of more marked rises of CIC in severe disease could, in fact, reflect increased tissue deposition with resultant intestinal injury. Alternatively, an increase in antigen formation or release occurring with severe disease may result in marked antigen excess and thus the formation of complexes which are poorly detected by indirect assays. ${ }^{13} 14$

Patients with CIC in our study did not have a higher incidence of extraintestinal manifestations than IBD patients without such complexes, in contrast to a recent report. ${ }^{3}$ Although differences in the populations of complexes measured by the different assays may be responsible for the discrepant findings, it is equally likely that there was a difference in the populations studied. Many of the patients with extraintestinal complications reported by Hodgson et al. $^{3}$ had liver disease. As various forms of liver disease result in immune complex production, 11141819 their data may reflect the production of liver disease associated complexes, rather than inflammatory bowel disease related CIC. Regardless of the cause of the above disparity, the absence of CIC in 46 per cent of the Hodgson et al. patients and 73 per cent of our patients with extraintestinal disease, indicates that the immune complexes measured are probably not the only factors associated with extraintestinal manifestations.

In recent work, published during the preparation of this manuscript, Soltis et al..$^{20}$ were unable to detect similar rises in circulating immune complexes in patients with chronic inflammatory bowel disease despite the use of several assay systems. They concluded that the 'raised CIC levels' detected by other groups using similar assays might be due to immunoglobulin aggregation produced during heat inactivation of complement or to the presence of low molecular weight C-reactive protein. Such material, however, does not appear to be responsible for the Raji reactivity noted in the present study. First, sera were not decomplemented for measurement in the Raji assay. Additionally, all sera were stored for siiort periods at $-70^{\circ} \mathrm{C}$ until their single thaw immediately before assay. Second, sucrose density gradient centrifugation of Raji positive IBD serum demonstrated reactivity in fractions containing materials of molecular weight greater than $7 \mathrm{~S}$. The levels noted in this study, therefore, appear to reflect in vivo CIC. The disparity between our results and those noted above probably arises from the relative sensitivites and specificities of the assays employed.

In conclusion, the $\mathrm{CIC}$ detected in patients with IBD appear to be related specifically to IBD and not simply to the destruction of the intestinal mucosal barrier. At present, the pathogenetic significance of these complexes is unknown. They may be of importance in the interrelationship of the activated cellular and humoral states in IBD. The immune complexes may specificially activate and direct lymphocyte cytotoxicity against intestinal cells possessing antigens identical with those contained within the complex. ${ }^{2122}$ Alternatively, the complexes may inhibit cellular responses directed at infected cells in a manner similar to the blocking phenomena or antibody modulation believed to occur with malignancy. ${ }^{23}$ Even if $\mathrm{CIC}$ are disease-related epiphenomena, without direct pathogenic effects, isolation and characterisation of the complexes and their constituents may help to identify disease specific antigen(s) and provide new insight into the aetiology and pathogenesis of IBD.

The authors would like to acknowledge the excellent technical assistance of Kathy O'Loughlin and Nancy Neyhard and to thank the surgical staff of the Massachusetts General Hospital whose cooperation made this study possible.

\section{References}

${ }^{1}$ Jewell DP, MacLennan ICM. Circulating immune complexes in inflammatory bowel. disease Clin Exp Immunol 1973; 14: 219-26.

${ }^{2}$ Doe WF, Booth CC, Brown DL. Evidence for complement-binding immune complexes in adult coeliac disease, and ulcerative colitis. Lancet 1973; 1: 402-3.

${ }^{3}$ Hodgson HJF, Potter BJ, Jewell DP. Immune complexes in ulcerative colitis and Crohn's disease. Clin Exp Immunol 1977; 29: 187-96.

${ }^{4}$ Nielsen H, Binder V, Daugharty H, Svehag SE. Circulating immune complexes in ulcerative colitis. I. Correlation to disease activity. Clin Exp Immunol 1978; 31: 72-80.

${ }^{5}$ Nielsen H, Hyltoft Petersen P, Svehag SE. Circulating immune complexes in ulcerative colitis. II. Correlation with serum protein concentrations and complement conversion products. Clin Exp Immunol 1978; 31 : 81-91. 
${ }^{6}$ Fiasse R, Lurhuma AZ, Cambiaso CL, Masson PL, Dive C. Circulating immune complexes and disease activity in Crohn's disease. Gut 1978; 19: 611-17.

'Truelove SC, Witts LJ. Cortisone in ulcerative colitis: final report on a therapeutic trial. $B r \operatorname{Med} J$ 1955; 2: 1041-48.

${ }^{8}$ de Dombal FT, Burton IL, Clamp SE, Goligher JC. Short-term course and prognosis of Crohn's disease. Gut 1974; 15: 435-43.

${ }^{9}$ Bartlett JG, Moon N, Chang TW, Taylor N, Onderdonk AB. Role of Clostridium difficile in antibiotic-associated pseudomembranous colitis. Gastroenterology 1978; 75: 778-82.

${ }^{10}$ Kemler BJ, Franklin WD, Alpert E, Bloch KJ. Failure to detect circulating immune complexes in allergic patients on injection therapy. Clin Allergy 1979; 9: 473-478.

${ }^{11}$ Wands JR, Dienstag JL, Bhan AK, Feller ER, Isselbacher KJ. Circulating immune complexes and complement activation in primary biliary cirrhosis. $N$ Engl J Med 1978; 298: 233-37.

${ }^{12}$ Rossen RD, Reisberg MA, Singer DB, et al. Soluble immune complexes in sera of patients with nephritis. Kidney Int 1976; 10: 256-63.

${ }^{13}$ Dixon FJ. The role of antigen-antibody complexes in disease. Harvey Lect 1962; 58: 21-52.

${ }^{14}$ Theofilopoulos AN, Wilson CB, Dixon FJ. The Raji cell radioimmune assay for detecting immune complexes in human sera. J Clin Invest 1976; 57: 169-82.

${ }^{15}$ Casali P, Bossus A, Carpentier NA, Lambert PH. Solid-phase enzyme immunoassay or radioimmunoassay for the detection of immune complexes based on their recognition by conglutinin: conglutinin-binding test. A comparative study with ${ }^{125}$ I-labelled $\mathrm{C}_{1} \mathrm{q}$ binding and Raji-cell RIA tests. Clin Exp Immunol 1977; 29: 342-54.
${ }^{16}$ Lambert PH, Dixon FJ, Zubler RH, et al. A WHO collaborative study for the evaluation of 18 methods for detecting immune complexes in serum. J Clin Lab Immunol 1978; 1: 1-15.

${ }^{17}$ Hodgson HJF, Potter BJ, Skinner J, Jewell DP. Immune-complex mediated colitis in rabbits. An experimental model. Gut 1978; 19: 225-32.

${ }^{18}$ Nydegger UE, Lambert PH, Gerber H, Miescher, PA. Circulating immune complexes in the serum in systemic lupus erythematosus and in carriers of hepatitis B antigen. Quantitation by binding to radiolabeled $C_{1} q$. J Clin Invest 1974; 54: 297-309.

${ }^{19}$ Wands JR, Mann E, Alpert E, Isselbacher KJ. The pathogenesis of arthritis associated with acute hepatitis-B surface antigen-positive hepatitis. Complement activation and characterisation of circulating immune complexes. J Clin Invest 1975; 55: 930-36.

${ }^{20}$ Soltis RD, Hasz D, Morris MJ, Wilson ID. Evidence against the presence of circulating immune complexes in chronic inflammatory bowel disease. Gastroenterology 1979; 76: 1380-85.

${ }^{21}$ Stobo JD, Tomasi TB, Huizenga KA, Spencer RJ, Shorter RG. In vitro studies of inflammatory bowel disease. Surface receptors of the mononuclear cell required to lyse allogeneic colon epithelial cells. Gastroenterology 1976; 70: 171-76.

${ }^{22}$ Kemler BJ, Alpert E. Immunopathogenesis of inflammatory bowel disease: studies of cytotoxicity of isolated human colon epithelial cells. Clin Rearch 1979; 27: 455A.

${ }^{23}$ Sjögren HO, Hellström I, Bansal SC, Hellström KE. Suggestive evidence that the 'blocking antibodies' of tumour-bearing individuals may be antigen-antibody complexes. Proc Natl Acad Sci USA 1971; 68: 1372-75. 